\title{
Every generalized quadrangle of order 5 having a regular point is symplectic
}

\author{
Bart De Bruyn \\ Ghent University, Department of Mathematics, Krijgslaan 281 (S22), B-9000 Gent, Belgium, \\ E-mail: bdb@cage.ugent.be
}

\begin{abstract}
For already many years now one of the most important open problems in the theory of generalized quadrangles is whether other classes of generalized quadrangles exist besides those that are currently known. This paper reports on an unsuccessful attempt to construct a new generalized quadrangle. As a by-product of our attempt, we however obtain the following new characterization result: every generalized quadrangle of order 5 that has at least one regular point is isomorphic to the quadrangle $W(5)$ arising from a symplectic polarity of $\mathrm{PG}(3,5)$. During the classification process we used the computer algebra system GAP to do certain computations or to experiment about an optimal strategy for the proof.
\end{abstract}

Keywords: generalized quadrangle, regular point, (generalized) admissible triple, affine plane MSC2010: 05B25, 51E12

\section{Introduction}

A partial linear space $\mathcal{Q}$ is called a generalized quadrangle of order $(s, t)$ for some $s, t \in$ $\mathbb{N}^{*}:=\mathbb{N} \backslash\{0\}$ if the following three properties hold:

(1) every line is incident with precisely $s+1$ points;

(2) every point is incident with precisely $t+1$ lines;

(3) for every line $L$ and every point $x$ not incident with $L$, there exists a unique point on $L$ collinear with $x$.

If $s=t$, then the generalized quadrangle $\mathcal{Q}$ is also said to be of order $s$. Generalized quadrangles were introduced by Jacques Tits in [19].

Suppose $\mathcal{Q}$ is a generalized quadrangle of order $(s, t)$ with point set $\mathcal{P}$. For every point $x$ of $\mathcal{Q}$, we denote by $x^{\perp}$ the set of all points of $\mathcal{Q}$ collinear with $x$ (including the point $x$ itself). If $X$ is a set of points of $\mathcal{Q}$, then we define $X^{\perp}:=\bigcap_{x \in X} x^{\perp}$ and $X^{\perp \perp}:=\left(X^{\perp}\right)^{\perp}$, 
hereby following the convention that $\emptyset^{\perp}=\mathcal{P}$. A hyperbolic line of $\mathcal{Q}$ is any set of the form $\{x, y\}^{\perp \perp}$ where $x$ and $y$ are two noncollinear points of $\mathcal{Q}$. Every hyperbolic line of $\mathcal{Q}$ has size at most $t+1$. If all hyperbolic lines through a point $x$ of $\mathcal{Q}$ have size $t+1$, then $x$ is called a regular point.

Let $\zeta$ be a symplectic polarity of $\mathrm{PG}(3, q)$. Let $W(q)$ denote the subgeometry of $\mathrm{PG}(3, q)$ defined by those points and lines of $\mathrm{PG}(3, q)$ that are totally isotropic with respect to $\zeta$. Then $W(q)$ is a generalized quadrangle of order $q$ all whose points are regular. The generalized quadrangle $W(q)$ is called a symplectic quadrangle. The following is the main result of this paper.

Theorem 1.1 Every generalized quadrangle of order 5 having at least one regular point is symplectic.

As Theorem 1.1 indicates, the current paper is concerned with the problem of classifying all GQ's of order $(s, t)$ for some specific values of $s$ and $t$. In the literature, a complete classification of all GQ's of order $(s, t)$ has been obtained whenever $s \in\{1,2,3\}$, $t \in\{1,2,3\}$ or $(s, t)=(4,4)$, see $[8,13,14,15]$. The GQ's with the smallest number of points for which the mentioned classification problem is still unsolved are those for which the order is equal to $(4,6)$ or $(5,5)$ (respectively corresponding to 125 and 156 points). Theorem 1.1 gives a complete classification of those GQ's of order $(5,5)$ that contain at least one regular point. Theorem 1.1 is equivalent with the statement that every generalized quadrangle of order $(4,6)$ having a regular spread is isomorphic to the GQ $A S(5)$ (see Section 2.1 for definitions).

Theorem 1.1 is also concerned with the problem of characterizing symplectic GQ's by means of suitable sets of regular points. One of the earliest combinatorial characterizations of GQ's $([2,16,17],[15,5.2 .1])$ states that every GQ of order $s \geq 2$ for which every point is regular is symplectic (and so $s$ is a prime power). In [5], the author showed that a GQ of order $s \geq 2$ is symplectic as soon as there is some hyperbolic line that entirely consists of regular points. Other characterizations of symplectic GQ's in terms of suitable sets of regular points can be found in [15] (see Theorems 1.3.6, 5.2.5 and 5.2.6). In [18, Theorem $4.3]$, it was shown that a generalized quadrangle of order $s \geq 2$ is symplectic as soon as there are two noncollinear regular points $x$ and $y$ such that the projective plane $\pi_{x}$ (as defined in Section 2.1) is Desarguesian. This implies that every generalized quadrangle of order 5 is symplectic as soon as there are two noncollinear regular points ([18, Corollary 4.4]). Theorem 1.1 improves this by saying that the existence of one regular point is already sufficient. Note that this cannot be further improved. The GQ $Q(4,5)$, which is the point-line dual of $W(5)$, is nonisomorphic to $W(5)$ and has no regular points at all. In fact, there is a conjecture in the area of generalized quadrangles which states that every GQ of order 5 is isomorphic to either $W(5)$ or $Q(4,5)$. 


\section{Preliminaries}

\subsection{Further notions regarding GQ's}

Let $\mathcal{Q}$ be a generalized quadrangle (GQ) of order $(s, t)$, where $s, t \in \mathbb{N}^{*}$. If $L_{1}$ and $L_{2}$ are two disjoint lines of $\mathcal{Q}$, then $\left\{L_{1}, L_{2}\right\}^{\perp}$ denotes the set of all lines meeting $L_{1}$ and $L_{2}$, and $\left\{L_{1}, L_{2}\right\}^{\perp \perp}$ denotes the set of all lines meeting every line of $\left\{L_{1}, L_{2}\right\}^{\perp}$. A spread of $\mathcal{Q}$ is a set $S$ of lines having the property that every point of $\mathcal{Q}$ is incident with a unique line of $S$. A spread $S$ of $\mathcal{Q}$ is called regular if for every two distinct lines $L_{1}, L_{2} \in S$, the set $\left\{L_{1}, L_{2}\right\}^{\perp \perp}$ has size $s+1$ and is contained in $S$. A spread $S$ is called a spread of symmetry if for every line $L \in S$ and all $l_{1}, l_{2} \in L$, there exists an automorphism of $\mathcal{Q}$ fixing each line of $S$ and mapping $l_{1}$ to $l_{2}$. Every spread of symmetry is a regular spread. If $S$ is a spread of a GQ of order $(s, t)$ with $t \neq 1$, then by [3, Theorem 4.1]), there are at most $s+1$ automorphisms of $\mathcal{Q}$ that fix each line of $S$, and equality holds if and only if $S$ is a spread of symmetry.

If $x$ is a point of a GQ $\mathcal{Q}$ of order $(s, t)$ with $s>1$, then there are at most $t$ automorphisms of $\mathcal{Q}$ that fix each point of $x^{\perp}$, see [15, 8.1]. If there are precisely $t$ such automorphisms, then $x$ is called a center of symmetry. Every center of symmetry is a regular point.

If $x$ is a regular point of a GQ of order $s \geq 2$, then the geometry defined on $x^{\perp}$ by the sets $\left\{y_{1}, y_{2}\right\}^{\perp \perp}$ where $y_{1}$ and $y_{2}$ are two distinct points of $x^{\perp}$ is a projective plane $\pi_{x}$ of order $s$, see e.g. [15, 1.3.1].

From every GQ $\mathcal{Q}$ of order $s>1$ having a regular point $x$, a GQ $\mathcal{P}(\mathcal{Q}, x)$ of order $(s-1, s+1)$ can be derived, see [11, Theorem 5.1] or [15, 3.1.4]. This new GQ is called the expansion of $\mathcal{Q}$ about $x$. The points of $\mathcal{P}(\mathcal{Q}, x)$ are the points of $\mathcal{Q}$ noncollinear with $x$ and the lines of $\mathcal{P}(\mathcal{Q}, x)$ are on the one hand the lines of $\mathcal{Q}$ nonincident with $x$ and on the other hand the hyperbolic lines through $x$ (natural incidence). The set $S(\mathcal{Q}, x)$ of all hyperbolic lines through $x$ is a regular spread of $\mathcal{P}(\mathcal{Q}, x)$. By De Soete and Thas $[7$, Theorems 2.7 and 2.8], this regular spread is a spread of symmetry if and only if $x$ is a center of symmetry. By [12, Theorem 1.1], for every GQ $\mathcal{Q}^{\prime}$ of order $(s-1, s+1), s \geq 2$ having a regular spread, there exists a generalized quadrangle $\mathcal{Q}$ of order $s$ and a regular point $x$ in $\mathcal{Q}$ such that $\mathcal{Q}^{\prime} \cong \mathcal{P}(\mathcal{Q}, x)$.

As told before, all points of the symplectic GQ $W(q)$ are regular. As the automorphism group of $W(q)$ is point-transitive there is essentially one GQ that arises by expanding $W(q)$ about one of its points. This GQ, which we denote here by $A S(q)$, was first constructed by Ahrens and Szekeres [1] and (only for $q$ even) by Hall [10].

\subsection{Generalized admissible triples}

Definition 2.1 Let $s, t \in \mathbb{N}^{*}$. A Steiner system of type $S(2, s+1$, st +1$)$ is a point-line geometry $(\mathcal{P}, \mathcal{B})$ having precisely $s t+1$ points, $s+1$ points on each line and a unique line through every pair of distinct points. Every point of such a Steiner system is incident with precisely $t$ lines. 
Definition 2.2 A generalized admissible triple (GAT for short) is a triple $\mathcal{T}=(\mathcal{S}, X, \Delta)$ for which:

- $X$ is a finite set whose size $s+1:=|X|$ is at least 2 .

- $\mathcal{S}$ is a Steiner system of type $S(2, s+1, s t+1)$ for some $t \in \mathbb{N}^{*}$.

- If $\mathcal{P}$ denotes the point set of $\mathcal{S}$, then $\Delta$ is a map from $\mathcal{P} \times \mathcal{P}$ to $\operatorname{Sym}(X)$ satisfying the following:

(GAT1) if $p, q$ and $r$ are collinear, then $\Delta(p, q) \cdot \Delta(q, r)=\Delta(p, r)$;

(GAT2) if $p, q$ and $r$ are not collinear, then the permutation $\Delta(p, q) \cdot \Delta(q, r) \cdot \Delta(r, p)$ has no fixpoints.

Above, $\operatorname{Sym}(X)$ denotes the group of all permutations of $X$ and we follow the convention here that $x^{\sigma_{1} \sigma_{2}}=\left(x^{\sigma_{1}}\right)^{\sigma_{2}}$ for all $x \in X$ and all $\sigma_{1}, \sigma_{2} \in \operatorname{Sym}(X)$. We denote the trivial permutation of $X$ by 1 . If $\mathcal{T}=(\mathcal{S}, X, \Delta)$ is a GAT, then by putting successively $q=p$ and $r=p$ in (GAT1), we see that $\Delta(p, p)=1$ and $\Delta(q, p)=\Delta(p, q)^{-1}$ for every two points $p$ and $q$ of $\mathcal{S}$. Generalized admissible triples were introduced in [5].

Proposition $2.3([5$, Theorem 7$])$ Let $\mathcal{S}_{1}=\left(\mathcal{P}_{1}, \mathcal{B}_{1}\right)$ and $\mathcal{S}_{2}=\left(\mathcal{P}_{2}, \mathcal{B}_{2}\right)$ be two isomorphic Steiner systems and $X_{1}, X_{2}$ two sets of the same cardinality. Let $\alpha$ be an isomorphism between $\mathcal{S}_{1}$ and $\mathcal{S}_{2}$ and for every point $p$ of $\mathcal{S}_{1}$ let $\theta_{p}$ be a bijection between $X_{1}$ and $X_{2}$. Suppose $\mathcal{T}_{1}=\left(\mathcal{S}_{1}, X_{1}, \Delta_{1}\right)$ is a GAT for a certain map $\Delta_{1}: \mathcal{P}_{1} \times \mathcal{P}_{1} \rightarrow \operatorname{Sym}\left(X_{1}\right)$. Then also $\mathcal{T}_{2}=\left(\mathcal{S}_{2}, X_{2}, \Delta_{2}\right)$ is a GAT, where $\Delta_{2}$ is the map from $\mathcal{P}_{2} \times \mathcal{P}_{2}$ to $\operatorname{Sym}\left(X_{2}\right)$ defined by

$$
\Delta_{2}\left(p^{\alpha}, q^{\alpha}\right):=\theta_{p}^{-1} \Delta_{1}(p, q) \theta_{q}, \quad \forall p, q \in \mathcal{P}_{1} .
$$

Definition 2.4 We say that two GAT's $\mathcal{T}_{1}=\left(\mathcal{S}_{1}, X_{1}, \Delta_{1}\right)$ and $\mathcal{T}_{2}=\left(\mathcal{S}_{2}, X_{2}, \Delta_{2}\right)$ are equivalent if there exists an isomorphism $\alpha$ from $\mathcal{S}_{1}$ to $\mathcal{S}_{2}$ and bijections $\theta_{p}: X_{1} \rightarrow X_{2}$ for points $p$ of $\mathcal{S}_{1}$ such that $\Delta_{2}\left(p^{\alpha}, q^{\alpha}\right)=\theta_{p}^{-1} \Delta_{1}(p, q) \theta_{q}$ for all points $p$ and $q$ of $\mathcal{S}_{1}$.

\subsection{GQ's from GAT's}

Proposition 2.5 ([5, Theorem 4]) Suppose $\mathcal{T}=(\mathcal{S}, X, \Delta)$ is a GAT where $\mathcal{S}=(\mathcal{P}, \mathcal{B})$ is a Steiner system of type $S(2, s+1$, st +1$)$. Let $\Gamma$ be the simple graph with vertex set $X \times \mathcal{P}$, where two distinct vertices $\left(x_{1}, p_{1}\right)$ and $\left(x_{2}, p_{2}\right)$ are adjacent whenever either

(a) $p_{1}=p_{2}$ and $x_{1} \neq x_{2}$, or

(b) $p_{1} \neq p_{2}$ and $x_{2}=x_{1}^{\Delta\left(p_{1}, p_{2}\right)}$.

Then $\Gamma$ is the collinearity graph of a $G Q \mathcal{Q}_{\mathcal{T}}$ of order $(s, t)$. For every point $p$ of $\mathcal{S}$, the set $L_{p}:=\{(x, p) \mid x \in X\}$ is a line of $\mathcal{Q}_{\mathcal{T}}$ and the set $S_{\mathcal{T}}$ of all lines obtained in this way is a regular spread of $\mathcal{Q}_{\mathcal{T}}$. 
Definition 2.6 For every GAT $\mathcal{T}$, we define $\Omega(\mathcal{T}):=\left(\mathcal{Q}_{\mathcal{T}}, S_{\mathcal{T}}\right)$, where $\mathcal{Q}_{\mathcal{T}}$ and $S_{\mathcal{T}}$ are as in Proposition 2.5.

Definition 2.7 Let $\mathcal{Q}_{1}$ and $\mathcal{Q}_{2}$ be two generalized quadrangles and let $S_{i}, i \in\{1,2\}$, be a regular spread of $\mathcal{Q}_{i}$. Then $\left(\mathcal{Q}_{1}, S_{1}\right)$ and $\left(\mathcal{Q}_{2}, S_{2}\right)$ are said to be equivalent if there exists an isomorphism from $\mathcal{Q}_{1}$ to $\mathcal{Q}_{2}$ mapping $S_{1}$ to $S_{2}$.

Proposition 2.8 ([5, Theorem 6]) Let $\mathcal{Q}$ be a generalized quadrangle of order $(s, t)$ having a regular spread $S$. Then there exists a $G A T \mathcal{T}$ such that $\Omega(T)$ is equivalent with $(\mathcal{Q}, S)$.

Proposition 2.9 ([5, Theorem 8]) Let $\mathcal{T}_{1}$ and $\mathcal{T}_{2}$ be two GAT's. Then $\Omega\left(\mathcal{T}_{1}\right)$ and $\Omega\left(\mathcal{T}_{2}\right)$ are equivalent if and only if $\mathcal{T}_{1}$ and $\mathcal{T}_{2}$ are equivalent.

\subsection{Admissible triples}

Definition 2.10 A GAT $\mathcal{T}=(\mathcal{S}, X, \Delta)$ is called an admissible triple (AT for short) if there exists a binary operation $\cdot$ on $X$ such that

$(\mathrm{AT} 1)(X, \cdot)$ is a group;

(AT2) $x^{-1} \cdot x^{\Delta\left(p_{1}, p_{2}\right)}$ only depends on the points $p_{1}$ and $p_{2}$ of $\mathcal{S}$ and not on the element $x$ of $X$.

Now, let $G$ denote the subgroup of $\operatorname{Sym}(X)$ consisting of the elements $\sigma_{y}, y \in X$, where each $\sigma_{y}$ maps the element $x \in X$ to the element $x \cdot y \in X$. If $p_{1}$ and $p_{2}$ are two points of $\mathcal{S}$, then putting $x_{p_{1}, p_{2}}:=x^{-1} \cdot x^{\Delta\left(p_{1}, p_{2}\right)}$ (with $x \in X$ arbitrary), we see that $x^{\Delta\left(p_{1}, p_{2}\right)}=x \cdot x_{p_{1}, p_{2}}$ for every $x \in X$, showing that $\Delta\left(p_{1}, p_{2}\right) \in G$. The conditions (GAT1) and (GAT2) can thus be replaced by the following condition: three points $p, q$ and $r$ of $\mathcal{S}$ are collinear if and only if $x_{p q} \cdot x_{q r}=x_{p r}$. This shows that the definition of AT as given here is equivalent with the definition of admissible triple as given in [3]. Indeed, in [3] this notion was defined as a triple $(\mathcal{S}, G, \Delta)$ satisfying:

- $G$ is a nontrivial finite group;

- $\mathcal{S}$ is a Steiner system of type $S(2, s+1, s t+1)$ where $s:=|G|-1$ and $t \in \mathbb{N}^{*}$;

- if $\mathcal{P}$ denotes the point set of $\mathcal{S}$, then $\Delta$ is a map from $\mathcal{P} \times \mathcal{P}$ to $G$ such that if $x, y, z \in \mathcal{P}$, then $x, y, z$ are incident with the same line of $\mathcal{S}$ if and only if $\Delta(x, y)$. $\Delta(y, z)=\Delta(x, z)$.

We end this subsection with stating two results that we will need to invoke later.

Proposition 2.11 ([5, Theorems 11 and 12]) Let $\mathcal{T}=(\mathcal{S}, X, \Delta)$ be a GAT, where the Steiner system $\mathcal{S}$ is not a line. Then $\mathcal{T}$ is a $A T$ if and only if $\operatorname{Im}(\Delta)$ generates a subgroup of size $|X|$ of $\operatorname{Sym}(X)$.

Proposition 2.12 ([3, Theorem 3.2]) If $\mathcal{T}=(\mathcal{S}, X, \Delta)$ is an AT, then the spread $S_{\mathcal{T}}$ of $\mathcal{Q}_{\mathcal{T}}$ is a spread of symmetry. 


\section{Proof of Theorem 1.1}

\subsection{Sets of permutations of $\{1,2,3,4,5\}$ satisfying Property $(*)$}

Definition 3.1 Let $\Sigma$ be a set of permutations of $X:=\{1,2,3,4,5\}$. We say that $\Sigma$ satisfies Property (*) if the following hold:

- $|\Sigma|=4$

- no element of $\Sigma$ has fixpoints;

- no permutation $\sigma_{1} \sigma_{2}^{-1}$ has fixpoints, where $\sigma_{1}, \sigma_{2}$ are two distinct elements of $\Sigma$.

Note that if $\sigma_{1} \sigma_{2}^{-1}$ has fixpoints, then each of the permutations $\sigma_{2} \sigma_{1}^{-1}, \sigma_{1}^{-1} \sigma_{2}, \sigma_{2}^{-1} \sigma_{1}$ also has fixpoints. If $\Sigma$ is a set of permutations of $X$ satisfying Property $(*)$, then for every $\sigma \in \operatorname{Sym}(X)$ also $\Sigma^{\sigma}:=\left\{\sigma^{-1} \sigma^{\prime} \sigma \mid \sigma^{\prime} \in \Sigma\right\}$ satisfies Property $(*)$. We call $\Sigma^{\sigma}$ the conjugation of $\Sigma$ by $\sigma$.

Lemma 3.2 Up to conjugation, there are precisely three sets of permutations of $X$ satisfying Property (*). These three sets are:

(1) $\Sigma_{1}^{*}:=\{(12345),(13524),(14253),(15432)\}$;

(2) $\Sigma_{2}^{*}:=\{(123)(45),(135)(24),(253)(14),(152)(34)\}$;

(3) $\Sigma_{3}^{*}:=\{(12345),(13)(254),(14352),(15324)\}$.

Proof. Put $\Sigma=\left\{\sigma_{1}, \sigma_{2}, \sigma_{3}, \sigma_{4}\right\}$. Since no $\sigma_{i}$ has fixpoints, each $\sigma_{i}$ has shape $(*, *, *, *, *)$ or $(*, *)(*, *, *)$. Observe that the sets $\Sigma_{1}^{*}, \Sigma_{2}^{*}, \Sigma_{3}^{*}$ defined above are mutually nonconjugate as they contain a different amount of cycles of length 5 (4, 0 and 3 , respectively). We will consider three cases.

(1) Suppose $\sigma_{1}, \sigma_{2}, \sigma_{3}$ and $\sigma_{4}$ are all cycles of length 5 . As we classify sets of permutations up to conjugacy, we may without loss of generality suppose that $\sigma_{1}=(12345)$ and that $\sigma_{2}, \sigma_{3}, \sigma_{4}$ are of the following form: $\sigma_{2}=(13 * * *), \sigma_{3}=(14 * * *), \sigma_{4}=(15 * * *)$. The fact that $\sigma_{2} \sigma_{1}^{-1}$ has no fixpoints implies that $\left\{\sigma_{1}, \sigma_{2}\right\}$ is equal to either $\{(12345),(13254)\}$, $\{(12345),(13524)\}$ or $\{(12345),(13542)\}$. Together with the facts that also $\sigma_{3} \sigma_{1}^{-1}$ and $\sigma_{3} \sigma_{2}^{-1}$ have no fixpoints, we see that $\left\{\sigma_{1}, \sigma_{2}, \sigma_{3}\right\}$ is equal to $\{(12345),(13254),(14352)\}$ or $\{(12345),(13524),(14253)\}$. If we now try to find $\sigma_{4}$ such that none of $\sigma_{4} \sigma_{1}^{-1}, \sigma_{4} \sigma_{2}^{-1}$, $\sigma_{4} \sigma_{3}^{-1}$ has fixpoints, we see that one possibility remains: $\sigma_{1}=(12345), \sigma_{2}=(13524)$, $\sigma_{3}=(14253)$ and $\sigma_{4}=(15432)$.

(2) Suppose none of $\sigma_{1}, \sigma_{2}, \sigma_{3}, \sigma_{4}$ is a cycle of length 5 . Then without loss of generality we may suppose that $\sigma_{1}=(123)(45), 1^{\sigma_{2}}=3,1^{\sigma_{3}}=4$ and $1^{\sigma_{4}}=5$. If $\sigma_{2}$ is of the form $(13)(*, *, *)$, then $\sigma_{2} \sigma_{1}^{-1}$ would fix 3 , which is impossible. So, $\sigma_{2}$ is of the form $(13 *)(* *)$. As $\sigma_{2} \sigma_{1}^{-1}$ has no fixpoints, $\sigma_{2} \neq(132)(45)$. Using the remaining freedom (given by the fact that we classify up to conjugacy), we may suppose that $\sigma_{2}=(135)(24)$. Then $\sigma_{3}$ 
and $\sigma_{4}$ are uniquely determined by the fact that $\left\{\sigma_{1}, \sigma_{2}, \sigma_{3}, \sigma_{4}\right\}$ satisfies Property $(*)$. We should have $\sigma_{3}=(14)(253)$ and $\sigma_{4}=(152)(34)$.

(3) Suppose that among $\sigma_{1}, \sigma_{2}, \sigma_{3}, \sigma_{4}$, there are permutations of shape $(*, *, *, *, *)$ and permutations of shape $(*, *, *)(*, *)$. Without loss of generality, we may suppose that $\sigma_{1}$ has shape $(*, *, *, *, *)$ and that $\sigma_{2}$ has shape $(*, *, *)(*, *)$. Since $\sigma_{2} \sigma_{1}^{-1}$ has no fixpoints, we may without loss of generality (we classify up to conjugacy) suppose that $\sigma_{1}=(12345)$, $\sigma_{2}=(13)(254), 1^{\sigma_{3}}=4$ and $1^{\sigma_{4}}=5$. The fact that $\left\{\sigma_{1}, \sigma_{2}, \sigma_{3}, \sigma_{4}\right\}$ satisfies Property $(*)$ now implies that $\sigma_{3}$ and $\sigma_{4}$ are uniquely determined. We should have $\sigma_{3}=(14352)$ and $\sigma_{4}=(15324)$.

Definition 3.3 Let $\mathcal{G}$ denote the set of all ordered quadruples $G=\left(\sigma_{1}, \sigma_{2}, \sigma_{3}, \sigma_{4}\right)$ for which $\widetilde{G}:=\left\{\sigma_{1}, \sigma_{2}, \sigma_{3}, \sigma_{4}\right\}$ is a set of four permutations satisfying Property $(*)$. We have $|\mathcal{G}|=1344$. Indeed, a straightforward argument shows that there are 144 elements $G \in \mathcal{G}$ for which $\widetilde{G}$ is conjugate with $\Sigma_{1}^{*}, 240$ elements $G \in \mathcal{G}$ for which $\widetilde{G}$ is conjugate with $\Sigma_{2}^{*}$ and 960 elements $G \in \mathcal{G}$ for which $\widetilde{G}$ is conjugate with $\Sigma_{3}^{*}$.

Definition 3.4 For every permutation $g \in \operatorname{Sym}(X)$ having no fixpoints, let $\mathcal{G}(g)$ denote the set of all $\left(\sigma_{1}, \sigma_{2}, \sigma_{3}, \sigma_{4}\right) \in \mathcal{G}$ for which $\sigma_{1}=g$. If $g$ has shape $(*, *, *, *, *)$, then $|\mathcal{G}(g)|=36$. If $g$ has shape $(*, *)(*, *, *)$, then $|\mathcal{G}(g)|=24$.

\subsection{Determination of all GQ's of order 5 with a regular point}

Suppose $\mathcal{Q}$ is a generalized quadrangle of order 5 having a regular point $x$. Put $\mathcal{Q}^{\prime}=$ $\mathcal{P}(\mathcal{Q}, x)$. Then $\mathcal{Q}^{\prime}$ is a GQ of order $(s, t)=(4,6)$ having a regular spread $S^{\prime}$ and so by Proposition 2.8 there exists a GAT $\mathcal{T}^{\prime}=\left(\mathcal{S}, X, \Delta^{\prime}\right)$ such that $\Omega\left(\mathcal{T}^{\prime}\right)$ is equivalent with $\left(\mathcal{Q}^{\prime}, S^{\prime}\right)$. As $|X|=5$, we may without loss of generality suppose that $X=$ $\{1,2,3,4,5\}$. The Steiner system $\mathcal{S}=(\mathcal{P}, \mathcal{B})$ has type $S(2, s+1, s t+1)=S(2,5,25)$ and hence is an affine plane of order 5 . Let $o$ be a fixed point of $\mathcal{S}$ and define $\Delta(x, y):=$ $\Delta^{\prime}(o, x) \Delta^{\prime}(x, y) \Delta^{\prime}(o, y)^{-1}$ for all points $x$ and $y$ of $\mathcal{S}$. By Proposition 2.3 and Definition $2.4, \mathcal{T}:=(\mathcal{S}, X, \Delta)$ is a GAT equivalent with $\mathcal{T}^{\prime}$. Hence $\Omega(\mathcal{T})$ is also equivalent with $\left(\mathcal{Q}^{\prime}, S^{\prime}\right)$ by Proposition 2.9. Observe also that $\Delta(o, x)=\Delta^{\prime}(o, o) \Delta^{\prime}(o, x) \Delta^{\prime}(o, x)^{-1}$ is the identical permutation of $X$ for every point $x$ of $\mathcal{S}$.

Lemma 3.5 Let $L$ be a line of $\mathcal{S}$ not containing o and let $x$ be a point of $L$. Then $\Sigma_{x, L}:=\{\Delta(x, y) \mid y \in L \backslash\{x\}\}$ is a set of four permutations of $X$ satisfying Property (*) and hence is conjugate to either $\Sigma_{1}^{*}, \Sigma_{2}^{*}$ or $\Sigma_{3}^{*}$.

Proof. If $y_{1}$ and $y_{2}$ are two distinct points of $L$, then $\Delta\left(y_{1}, y_{2}\right)=\Delta\left(o, y_{1}\right) \Delta\left(y_{1}, y_{2}\right) \Delta\left(y_{2}, o\right)$ has no fixpoints by (GAT2). Hence:

- $\Delta(x, y)$ has no fixpoints for every $y \in L \backslash\{x\}$.

- Since $\Delta\left(x, y_{1}\right)^{-1} \Delta\left(x, y_{2}\right)=\Delta\left(y_{1}, y_{2}\right)$ (by (GAT1)), $\Delta\left(x, y_{1}\right)$ and $\Delta\left(x, y_{2}\right)$ are distinct and also $\Delta\left(x, y_{1}\right) \Delta\left(x, y_{2}\right)^{-1}$ has no fixpoints. 
Lemma 3.6 Let $L$ be a line of $\mathcal{S}$ containing o and let $x$ be a point not on $L$. Then $\{\Delta(x, y) \mid y \in L \backslash\{o\}\}$ is a set of four permutations of $X$ satisfying Property (*) and hence is conjugate to either $\Sigma_{1}^{*}, \Sigma_{2}^{*}$ or $\Sigma_{3}^{*}$.

Proof. If $y \in L \backslash\{o\}$, then $\Delta(x, y)=\Delta(y, o) \Delta(o, x) \Delta(x, y)$ has no fixpoints by (GAT2). If $y_{1}$ and $y_{2}$ are two distinct points of $L \backslash\{o\}$, then $\Delta\left(y_{1}, y_{2}\right)=\Delta\left(y_{1}, o\right) \Delta\left(o, y_{2}\right)=1$ by (GAT1). Moreover, $\Delta\left(x, y_{1}\right) \Delta\left(y_{1}, y_{2}\right) \Delta\left(y_{2}, x\right)=\Delta\left(x, y_{1}\right) \Delta\left(x, y_{2}\right)^{-1}$ has no fixpoints by (GAT2).

Lemma 3.7 There are two possibilities for a line $L$ of $\mathcal{S}$ that does not contain o:

(I) For any point $x$ of $L$, we have that $\Sigma_{x, L} \cong \Sigma_{1}^{*}$.

(II) There exists a unique point $x$ on $L$ such that $\Sigma_{x, L} \cong \Sigma_{2}^{*}$. If $y$ is another point of $L$, then $\Sigma_{y, L} \cong \Sigma_{3}^{*}$.

Proof. Let $u$ be an arbitrary point of $L$. Observe that if we know all values $\Delta\left(u, u^{\prime}\right)$ where $u^{\prime} \in L$, then we also know all values $\Delta\left(u_{1}, u_{2}\right)$ where $u_{1}, u_{2} \in L$. Indeed, by (GAT1) we know that $\Delta\left(u_{1}, u_{2}\right)=\Delta\left(u, u_{1}\right)^{-1} \Delta\left(u, u_{2}\right)$. We distinguish three cases.

(1) Suppose $\Sigma_{u, L} \cong \Sigma_{1}^{*}$. Then without loss of generality we may suppose that $\Sigma_{u, L}=\Sigma_{1}^{*}=\{(12345),(13524),(14253),(15432)\}$. Using the above observation, one readily computes that also $\Sigma_{u^{\prime}, L}=\Sigma_{1}^{*}$ for every $u^{\prime} \in L \backslash\{u\}$.

(2) Suppose $\Sigma_{u, L} \cong \Sigma_{2}^{*}$. Then without loss of generality we may suppose that $\Sigma_{u, L}=$ $\Sigma_{2}^{*}=\{(123)(45),(135)(24),(253)(14),(152)(34)\}$. Let $u^{\prime}$ be an arbitrary point of $L \backslash\{u\}$. Using the above observation, one readily finds that $\Delta\left(u^{\prime}, u\right)$ has shape $(*, *, *)(*, *)$ and that $\Delta\left(u^{\prime}, u^{\prime \prime}\right)$ has shape $(*, *, *, *, *)$ for every $u^{\prime \prime} \in L \backslash\left\{u, u^{\prime}\right\}$. So, $\Sigma_{u^{\prime}, L} \cong \Sigma_{3}^{*}$ and $x=u$ is the unique point on $L$ for which $\Sigma_{x, L} \cong \Sigma_{2}^{*}$.

(3) If $\Sigma_{u, L} \cong \Sigma_{3}^{*}$, then without loss of generality we may suppose that $\Sigma_{u, L}=\Sigma_{3}^{*}=$ $\{(12345),(13)(254),(14352),(15324)\}$. If $u^{\prime}$ is the unique point of $L$ for which $\Delta\left(u, u^{\prime}\right)=$ (13)(254), then one computes that $\Sigma_{u^{\prime}, L}=\{(12)(354),(13)(245),(14)(253),(15)(234)\} \cong$ $\Sigma_{2}^{*}$. By part (2), we then know that $\Sigma_{u^{\prime \prime}, L} \cong \Sigma_{3}^{*}$ for every point $u^{\prime \prime} \in L \backslash\left\{u^{\prime}\right\}$.

Proposition 3.8 If all lines of $\mathcal{S}$ not containing o are of Type (I), then $\mathcal{Q} \cong W(5)$.

Proof. Consider a line $L$ of $\mathcal{S}$ not containing $o$, let $y_{1}, y_{2}$ be two distinct points on $L$ and define $\delta:=\Delta\left(y_{1}, y_{2}\right)$. Since $L$ is of Type (I), $\delta$ is a cycle of length 5 . For every $y \in \mathcal{P} \backslash\{o\}$, we define $\Sigma_{y}:=\{\Delta(y, z) \mid z \in \mathcal{P} \backslash$ oy $\}$. Then $\Sigma_{y}$ only consists of cycles of length 5 . By Lemmas 3.5 and 3.6, we respectively have

(a) if $\Delta(y, z) \in\left\{\delta, \delta^{2}, \delta^{3}, \delta^{4}\right\}$ for two distinct points $y$ and $z$ of $\mathcal{S}$, then $\Delta\left(y, z^{\prime}\right) \in$ $\left\{\delta, \delta^{2}, \delta^{3}, \delta^{4}\right\}$ for any point $z^{\prime} \in y z \backslash\{y\}$;

(b) if $\Delta(y, z) \in\left\{\delta, \delta^{2}, \delta^{3}, \delta^{4}\right\}$ for two distinct points $y$ and $z$ of $\mathcal{S}$, then $\Delta\left(y, z^{\prime}\right) \in$ $\left\{\delta, \delta^{2}, \delta^{3}, \delta^{4}\right\}$ for any point $z^{\prime} \in o z \backslash\{o\}$.

(Note that $o \notin y z$ since $\Delta(y, z) \neq 1$.) Properties (a) and (b) imply the following: 
(c) Let $y$ and $z$ be two distinct points of $\mathcal{S}$ such that $o \notin y z$, and let $K$ be the unique line through $o$ parallel to $y z$. If $\Delta(y, z) \in\left\{\delta, \delta^{2}, \delta^{3}, \delta^{4}\right\}$, then $\Delta\left(y, z^{\prime}\right) \in\left\{\delta, \delta^{2}, \delta^{3}, \delta^{4}\right\}$ for every $z^{\prime} \in \mathcal{P} \backslash($ oy $\cup K)$.

Let $y$ and $z$ be two arbitrary points of $\mathcal{S}$ such that $o \notin y z$ and $\Delta(y, z) \in\left\{\delta, \delta^{2}, \delta^{3}, \delta^{4}\right\}$, and let $z_{1}$ be an arbitrary point of $\mathcal{P} \backslash\{o\}$ such that $o z_{1}$ is parallel with $y z$. If $z_{2}$ is a point of $y z_{1}$ distinct from $y$ and $z_{1}$, then $\Delta\left(y, z_{2}\right) \in\left\{\delta, \delta^{2}, \delta^{3}, \delta^{4}\right\}$ by Property (c). Property (a) then implies that $\Delta\left(y, z_{1}\right) \in\left\{\delta, \delta^{2}, \delta^{3}, \delta^{4}\right\}$. So, we have:

(d) Let $y$ and $z$ be two distinct points of $\mathcal{S}$ such that $o \notin y z$. If $\Delta(y, z) \in\left\{\delta, \delta^{2}, \delta^{3}, \delta^{4}\right\}$, then $\Delta\left(y, z^{\prime}\right) \in\left\{\delta, \delta^{2}, \delta^{3}, \delta^{4}\right\}$ for every $z^{\prime} \in \mathcal{P} \backslash$ oy.

Since $\Delta\left(y_{1}, y_{2}\right)=\delta$, we thus have that:

(e) $\Delta\left(y_{1}, z\right) \in\left\{\delta, \delta^{2}, \delta^{3}, \delta^{4}\right\}$ for every $z \in \mathcal{P} \backslash o y_{1}$.

If $z \in \mathcal{P} \backslash o y_{1}$, then $\Delta\left(z, y_{1}\right)=\Delta\left(y_{1}, z\right)^{-1} \in\left\{\delta, \delta^{2}, \delta^{3}, \delta^{4}\right\}$. By Property (d) we can thus conclude that $\Delta\left(z, z^{\prime}\right) \in\left\{\delta, \delta^{2}, \delta^{3}, \delta^{4}\right\}$ for all $z, z^{\prime} \in \mathcal{P}$ for which $z \notin o y_{1}$ and $z^{\prime} \notin o z$. If $z \in o y_{1} \backslash\{o\}$ and $z^{\prime} \in \mathcal{P} \backslash o y_{1}$, then the latter fact implies that also $\Delta\left(z, z^{\prime}\right)=\Delta\left(z^{\prime}, z\right)^{-1} \in$ $\left\{\delta, \delta^{2}, \delta^{3}, \delta^{4}\right\}$. Summarizing, we thus have that:

(f) $\Delta(y, z) \in\left\{\delta, \delta^{2}, \delta^{3}, \delta^{4}\right\}$ for every two distinct points $y$ and $z$ of $\Delta$ for which $o \notin y z$.

This implies that:

(g) $\langle\operatorname{Im}(\Delta)\rangle=\langle\delta\rangle$ is a group of order 5 .

Since $\langle\operatorname{Im}(\Delta)\rangle$ is a group of order $5, \mathcal{T}$ must be an AT by Proposition 2.11. This implies by Proposition 2.12 that $S^{\prime}$ is a spread of symmetry of $\mathcal{Q}^{\prime}$. So, $x$ must be a center of symmetry of $\mathcal{Q}$ by [7, Theorem 2.7]. By [4] there exists up to isomorphism a unique generalized quadrangle of order 5 having a center of symmetry. We conclude that $\mathcal{Q}$ is isomorphic to $W(5)$.

In the sequel, we will suppose that there exists a line $L$ of Type (II) of $\mathcal{S}$ (not containing $o$ ). We denote by $x_{0}$ the unique point $x \in L$ for which $\Sigma_{x, L} \cong \Sigma_{2}^{*}$. We will show that this case cannot occur. However, we were only able to prove this by means of computer computations using the computer algebra system GAP [9] (see Lemma 3.13 and the discussion before Proposition 3.16).

Put $L=\left\{x_{0}, x_{1}, x_{2}, x_{3}, x_{4}\right\}$ and $o x_{i}=\left\{o, x_{i}, y_{i 1}, y_{i 2}, y_{i 3}\right\}$ for every $i \in\{0,1,2,3,4\}$. Let $\left\{o, z_{1}, z_{2}, z_{3}, z_{4}\right\}$ denote the unique line through $o$ parallel with $L$.

In view of Definition 2.4 and Proposition 2.9, we may without loss of generality suppose that

$$
\Sigma_{x_{0}, L}=\Sigma_{2}^{*}=\{(123)(45),(135)(24),(253)(14),(152)(34)\}
$$

and

$$
\Delta\left(x_{0}, x_{1}\right)=(123)(45), \quad \Delta\left(x_{0}, x_{2}\right)=(135)(24) .
$$


So, $\left(\Delta\left(x_{0}, x_{3}\right), \Delta\left(x_{0}, x_{4}\right)\right)$ is equal to either $((253)(14),(152)(34))$ or $((152)(34),(253)(14))$. We put $\epsilon:=+$ if the former case occurs and $\epsilon:=-$ if the latter case occurs. If we put

$$
\begin{aligned}
& g_{00}^{+}:=1, g_{01}^{+}:=(123)(45), g_{02}^{+}:=(135)(24), g_{03}^{+}:=(253)(14), g_{04}^{+}:=(152)(34), \\
& g_{00}^{-}:=1, g_{01}^{-}:=(123)(45), g_{02}^{-}:=(135)(24), g_{03}^{-}:=(152)(34), g_{04}^{-}:=(253)(14),
\end{aligned}
$$

and

$$
g_{i j}^{\epsilon}:=\left(g_{1 i}^{\epsilon}\right)^{-1} \cdot g_{1 j}^{\epsilon}
$$

for all $i, j \in\{0,1,2,3,4\}$ with $i \neq 0$, then (GAT1) implies that $\Delta\left(x_{i}, x_{j}\right)=g_{i j}^{\epsilon}$ for all $i, j \in\{0,1,2,3,4\}$.

Definition 3.9 Let $\mathcal{F}$ denote the set of all maps $f: \mathcal{P} \rightarrow \operatorname{Sym}(X)$. A map $f \in \mathcal{F}$ is called good with respect to a point $x \in \mathcal{P} \backslash\{o\}$ if for every line $K$ through $x$ distinct from $o x$ and any two distinct points $y_{1}, y_{2} \in K$, the permutation $f\left(y_{1}\right)^{-1} f\left(y_{2}\right)$ does not have fixpoints.

Definition 3.10 In this definition, we follow the convention that subindices are taken modulo 5 . For every $i \in\{0,1,2,3,4\}$,

$$
\pi_{i}^{\epsilon}: \mathcal{G}\left(g_{i, i+1}^{\epsilon}\right) \times \mathcal{G}\left(g_{i, i+2}^{\epsilon}\right) \times \mathcal{G}\left(g_{i, i+3}^{\epsilon}\right) \times \mathcal{G}\left(g_{i, i+4}^{\epsilon}\right) \times \mathcal{G} \rightarrow \mathcal{F}
$$

is the map which sends the element

$$
\begin{gathered}
\left(\left(g_{i, i+1}^{\epsilon}, \sigma_{1}^{(i+1)}, \sigma_{2}^{(i+1)}, \sigma_{3}^{(i+1)}\right),\left(g_{i, i+2}^{\epsilon}, \sigma_{1}^{(i+2)}, \sigma_{2}^{(i+2)}, \sigma_{3}^{(i+2)}\right),\left(g_{i, i+3}^{\epsilon}, \sigma_{1}^{(i+3)}, \sigma_{2}^{(i+3)}, \sigma_{3}^{(i+3)}\right),\right. \\
\left.\left(g_{i, i+4}^{\epsilon}, \sigma_{1}^{(i+4)}, \sigma_{2}^{(i+4)}, \sigma_{3}^{(i+4)}\right),\left(\sigma_{1}, \sigma_{2}, \sigma_{3}, \sigma_{4}\right)\right)
\end{gathered}
$$

to the map $f: \mathcal{P} \rightarrow \operatorname{Sym}(X)$ defined by

- $f(o)=f\left(y_{i 1}\right)=f\left(y_{i 2}\right)=f\left(y_{i 3}\right)=1$,

- $f\left(x_{j}\right)=g_{i j}^{\epsilon}$ for every $j \in\{0,1,2,3,4\}$,

- $f\left(y_{j k}\right)=\sigma_{k}^{(j)}$ for all $j \in\{0,1,2,3,4\} \backslash\{i\}$ and all $k \in\{1,2,3\}$,

- $f\left(z_{j}\right)=\sigma_{j}$ for every $j \in\{1,2,3,4\}$.

Definition 3.11 For every $i \in\{0,1,2,3,4\}$, let $\mathcal{A}_{i}^{\epsilon}$ denote the set of all $f \in \mathcal{F}$ belonging to the image of $\pi_{i}^{\epsilon}$ that are good with respect to $x_{i}$. For distinct $i, j \in\{0,1,2,3,4\}$, let $\mathcal{A}_{i j}^{\epsilon}$ denote the set of all pairs $\left(f_{i}, f_{j}\right)$ such that

- $f_{i} \in \mathcal{A}_{i}^{\epsilon}$ and $f_{j} \in \mathcal{A}_{j}^{\epsilon}$;

- for every point $x$ not on $L, f_{i}(x) \cdot\left(f_{j}(x)\right)^{-1} \cdot g_{j i}^{\epsilon}$ has no fixpoints. 
We have $\left(f_{i}, f_{j}\right) \in \mathcal{A}_{i j}^{\epsilon}$ if and only if $\left(f_{j}, f_{i}\right) \in \mathcal{A}_{j i}^{\epsilon}$. For $l \geq 3$ mutually distinct $i_{1}, i_{2}, \ldots, i_{l} \in\{0,1,2,3,4\}$, let $\mathcal{A}_{i_{1}, i_{2}, \ldots, i_{l}}^{\epsilon}$ denote the set of all $l$-tuples $\left(f_{i_{1}}, f_{i_{2}}, \ldots, f_{i_{l}}\right)$ such that $\left(f_{j}, f_{j^{\prime}}\right) \in \mathcal{A}_{j, j^{\prime}}^{\epsilon}$ for all $j, j^{\prime} \in\left\{i_{1}, i_{2}, \ldots, i_{l}\right\}$ with $j \neq j^{\prime}$. The importance of these sets follows from the following lemma.

Lemma 3.12 For every $i \in\{0,1,2,3,4\}$ and every $x \in \mathcal{P}$, we put $f_{i}(x):=\Delta\left(x_{i}, x\right)$. Then $\left(f_{0}, f_{1}, f_{2}, f_{3}, f_{4}\right) \in \mathcal{A}_{01234}^{\epsilon}$.

Proof. Let $i \in\{0,1,2,3,4\}$. Let $K$ be a line through $x_{i}$ distinct from $o x_{i}$ and let $y_{1}, y_{2}$ be two distinct points on $K$. Then $\Delta\left(y_{1}, y_{2}\right)=\Delta\left(o, y_{1}\right) \Delta\left(y_{1}, y_{2}\right) \Delta\left(y_{2}, o\right)$ has no fixpoints by (GAT2), implying that also $f_{i}\left(y_{1}\right)^{-1} f_{i}\left(y_{2}\right)=\Delta\left(y_{1}, x_{i}\right) \Delta\left(x_{i}, y_{2}\right)=\Delta\left(y_{1}, y_{2}\right)$ has no fixpoints. So, $f_{i}$ is good with respect to $x_{i}$. By Lemma 3.6 and the fact that $\Delta\left(x_{i}, x_{j}\right)=g_{i j}^{\epsilon}$ for all $i, j \in\{0,1,2,3,4\}, f_{i}$ belongs to the image of $\pi_{i}^{\epsilon}$. Hence, $f_{i} \in \mathcal{A}_{i}^{\epsilon}$.

By (GAT2), $f_{i}(x) \cdot f_{j}(x)^{-1} \cdot g_{j i}^{\epsilon}=\Delta\left(x_{i}, x\right) \Delta\left(x, x_{j}\right) \Delta\left(x_{j}, x_{i}\right)$ has no fixpoints for every point $x$ not on $L$ and every two distinct $i, j \in\{0,1,2,3,4\}$. So, $\left(f_{i}, f_{j}\right) \in \mathcal{A}_{i j}^{\epsilon}$ for any two distinct $i, j \in\{0,1,2,3,4\}$.

In the computer algebra system GAP, a model of the affine plane $\mathrm{AG}(2,5)$ with point set $\{1,2, \ldots, 25\}$ and line set lines can be implemented as follows:

AutGroup:=AllPrimitiveGroups (DegreeOperation, 25, Size, 12000) [1] ;

Stab:=Stabilizer (AutGroup, $[1,2]$, OnTuples) ;

$1:=$ Filtered (Orbits (Stab, [1..25]), $q->\operatorname{Size}(q)=1)$;

lines:=Orbit (AutGroup, Union (1 [1] , 1 [2] , 1 [3] , 1 [4] , 1 [5] ) , OnSets) ;

Using this model for $\mathrm{AG}(2,5)$, GAP told us that $\{1,2,3,4,5\}$ and $\{6,7,8,9,10\}$ are (necessarily parallel) lines. Also $\{1,11,16,21,6\},\{1,13,19,25,7\},\{1,14,20,22,8\},\{1,15,17,23$, $9\}$ and $\{1,12,18,24,10\}$ are lines. So, we can in fact make the following choices:

$$
\begin{gathered}
o=1, z_{1}=2, z_{2}=3, z_{3}=4, z_{4}=5, x_{0}=6, x_{1}=7, x_{2}=8, x_{3}=9, x_{4}=10, \\
\left(y_{01}, y_{02}, y_{03}\right)=(11,16,21),\left(y_{11}, y_{12}, y_{13}\right)=(13,19,25),\left(y_{21}, y_{22}, y_{23}\right)=(14,20,22), \\
\left(y_{31}, y_{32}, y_{33}\right)=(15,17,23),\left(y_{41}, y_{42}, y_{43}\right)=(12,18,24) .
\end{gathered}
$$

In GAP we can store the elements $f \in \mathcal{F}$ as arrays of length 25 whose $i$-th entry is the permutation of $\{1,2,3,4,5\}$ which is the image of the point $i$ under the map $f$. With these conventions in mind, we have implemented the sets $\mathcal{G}$ and $\mathcal{G}(g)$ and the maps $\pi_{i}^{\epsilon}$, which ultimately allowed us to compute the sets $\mathcal{A}_{i_{1}, i_{2}, \ldots, i_{l}}^{\epsilon}$, see [6]. Our results are summarized as follows.

Lemma 3.13 We have

- $\left|\mathcal{A}_{0}^{+}\right|=\left|\mathcal{A}_{0}^{-}\right|=75$ and $\left|\mathcal{A}_{i}^{+}\right|=\left|\mathcal{A}_{i}^{-}\right|=150$ for every $i \in\{1,2,3,4\}$;

- $\left|\mathcal{A}_{0 i}^{+}\right|=\left|\mathcal{A}_{0 i}^{-}\right|=291$ for every $i \in\{1,2,3,4\}$; 
- $\left|\mathcal{A}_{i j}^{+}\right|=\left|\mathcal{A}_{i j}^{-}\right| \in\{282,332\}$ for all $i, j \in\{1,2,3,4\}$ with $i \neq j$;

- $\left|\mathcal{A}_{012}^{+}\right|=\left|\mathcal{A}_{012}^{-}\right|=36$;

- $\left|\mathcal{A}_{0123}^{+}\right|=\left|\mathcal{A}_{0123}^{-}\right|=\left|\mathcal{A}_{01234}^{+}\right|=\left|\mathcal{A}_{01234}^{-}\right|=2$.

By Lemma 3.12 we know that with $\Delta$ there is associated an element of $\mathcal{A}_{01234}^{\epsilon}$. We now show in the following two lemmas, that $\Delta$ (if it exists) can be uniquely reconstructed from this element of $\mathcal{A}_{01234}^{\epsilon}$.

Lemma 3.14 Let $K=\left\{o, u_{1}, u_{2}, u_{3}, u_{4}\right\}$ be a line through o and let $v$ be a point not on $K$. Then $\Delta\left(v, u_{4}\right)$ is uniquely determined by $\Delta\left(v, u_{1}\right), \Delta\left(v, u_{2}\right)$ and $\Delta\left(v, u_{3}\right)$.

Proof. It suffices to show that $k^{\Delta\left(v, u_{4}\right)}$ is uniquely determined by $k^{\Delta\left(v, u_{1}\right)}, k^{\Delta\left(v, u_{2}\right)}$ and $k^{\Delta\left(v, u_{3}\right)}$ for every $k \in\{1,2,3,4,5\}$. The fact that $\Delta(o, v) \Delta\left(v, u_{i}\right) \Delta\left(u_{i}, o\right)=\Delta\left(v, u_{i}\right)$ and $\Delta\left(v, u_{i}\right) \Delta\left(u_{i}, u_{j}\right) \Delta\left(u_{j}, v\right)=\Delta\left(v, u_{i}\right) \Delta\left(v, u_{j}\right)^{-1}$ have no fixpoints if $i, j \in\{1,2,3,4\}$ with $i \neq j$ implies that $k^{\Delta\left(v, u_{4}\right)}$ is the unique element of $\{1,2,3,4,5\}$ distinct from $k, k^{\Delta\left(v, u_{1}\right)}$, $k^{\Delta\left(v, u_{2}\right)}$ and $k^{\Delta\left(v, u_{3}\right)}$.

Lemma 3.15 For every $i \in\{0,1,2,3,4\}$ and every $x \in \mathcal{P}$, put $f_{i}(x):=\Delta\left(x_{i}, x\right)$. Then $\Delta$ is uniquely determined by $\left(f_{0}, f_{1}, f_{2}, f_{3}, f_{4}\right) \in \mathcal{A}_{01234}^{\epsilon}$.

Proof. Let $y$ and $z$ be two points of $\mathcal{P}$. If $o, y$ and $z$ are collinear, then $\Delta(y, z)=1$. If $y \neq z$ and the line $y z$ contains a point $x_{i}$, then $\Delta(y, z)=\Delta\left(x_{i}, y\right)^{-1} \Delta\left(x_{i}, z\right)=f_{i}(y)^{-1} f_{i}(z)$.

Suppose $y \neq z$ and $y z$ is a line parallel with $L$, distinct from $L$ not containing $o$. Put $o z=\{o, z, u, v, w\}$. Since $\Delta(y, u), \Delta(y, v)$ and $\Delta(y, w)$ are uniquely determined by $\left(f_{0}, f_{1}, f_{2}, f_{3}, f_{4}\right)$ (see previous paragraph), also $\Delta(y, z)$ is uniquely determined by $\left(f_{0}, f_{1}, f_{2}, f_{3}, f_{4}\right)$ by Lemma 3.14 .

Since $\left|\mathcal{A}_{01234}^{\epsilon}\right|=2$, there are at most two possibilities for $\Delta$ for each $\epsilon \in\{+,-\}$. Based on the methods exposed in the proofs of Lemmas 3.14 and 3.15, we have implemented a computer program in GAP to reconstruct $\Delta$ from the possible elements of $\mathcal{A}_{01234}^{\epsilon}$, see [6]. For both $\epsilon=+$ and $\epsilon=-$, it turned out that none of the two elements of $\mathcal{A}_{01234}^{\epsilon}$ actually gives rise to a map $\Delta$ that satisfies (GAT1) and (GAT2). So, our assumption on the existence of lines of Type (II) was incorrect. We conclude:

Proposition 3.16 Every line of $\mathcal{S}$ not containing o has Type (I) and $\mathcal{Q}$ is isomorphic to $W(5)$.

\section{References}

[1] R. W. Ahrens and G. Szekeres. On a combinatorial generalization of 27 lines associated with a cubic surface. J. Austral. Math. Soc. 10 (1969), 485-492. 
[2] C. T. Benson. On the structure of generalized quadrangles. J. Algebra 15 (1970), 443-454.

[3] B. De Bruyn. Generalized quadrangles with a spread of symmetry. European J. Combin. 20 (1999), 759-771.

[4] B. De Bruyn. The uniqueness of the generalized quadrangle of order 5 with an axis of symmetry. Ars Combin. 88 (2008), 203-216.

[5] B. De Bruyn. A coordinatization structure for generalized quadrangles with a regular spread. European J. Combin. 29 (2008), 242-253.

[6] B. De Bruyn. GAP code for "Every generalized quadrangle of order 5 having a regular point is symplectic". See http://cage.ugent.be/ ^bdb.

[7] M. De Soete and J. A. Thas. A coordinatization of generalized quadrangles of order $(s, s+2)$. J. Combin. Theory Ser. A 48 (1988), 1-11.

[8] S. Dixmier and F. Zara. Etude d'un quadrangle généralisé autour de deux de ses point non liés. Preprint, 1976.

[9] The GAP Group, GAP - Groups, Algorithms, and Programming, Version 4.4.12; 2008. (http://www.gap-system.org)

[10] M. Hall, Jr. Affine generalized quadrilaterals. In Studies in Pure Mathematics. Academic Press, London, 1971, pp. 113-116.

[11] S. E. Payne. Nonisomorphic generalized quadrangles. J. Algebra 18 (1971), 201-212.

[12] S. E. Payne. The equivalence of certain generalized quadrangles. J. Combinatorial Theory Ser. A 10 (1971), 284-289.

[13] S. E. Payne. Generalized quadrangles of order 4. I. J. Combinatorial Theory Ser. A 22 (1977), 267-279.

[14] S. E. Payne. Generalized quadrangles of order 4. II. J. Combinatorial Theory Ser. A 22 (1977), 280-288.

[15] S. E. Payne and J. A. Thas. Finite generalized quadrangles. Second edition. EMS Series of Lectures in Mathematics. European Mathematical Society, Zürich, 2009.

[16] R. Singleton. On minimal graphs of maximum even girth. J. Combinatorial Theory 1 (1966), 306-332.

[17] G. Tallini. Strutture di incidenza dotate di polarità. Rend. Sem. Mat. Fis. Milano 41 (1971), 75-113. 
[18] J. A. Thas. A characterization of the generalized quadrangle $W(q)$ and applications. Trends in incidence and Galois geometries: a tribute to Giuseppe Tallini, 299-310, Quad. Mat., 19, Dept. Math., Seconda Univ. Napoli, Caserta, 2009.

[19] J. Tits. Sur la trialité et certains groupes qui s'en déduisent. (French) Inst. Hautes Etudes Sci. Publ. Math. 1959, no. 2, 13-60. 